\title{
Erratum to: Developments in Time-Division Multiplexing of X-ray Transition-Edge Sensors
}

\author{
W. B. Doriese ${ }^{1}$ - K. M. Morgan' ${ }^{1}$ - D. A. Bennett ${ }^{1}$ - E. V. Denison ${ }^{1}$. \\ C. P. Fitzgerald ${ }^{1}$ - J. W. Fowler ${ }^{1}$ - J. D. Gard ${ }^{1}$ - J. P. Hays-Wehle ${ }^{1}$. \\ G. C. Hilton ${ }^{1}$ - K. D. Irwin ${ }^{2}$ - Y. I. Joe ${ }^{1}$ - J. A. B. Mates ${ }^{1}$ - G. C. O'Neil ${ }^{1}$. \\ C. D. Reintsema ${ }^{1}$ - N. O. Robbins ${ }^{1}$ - D. R. Schmidt ${ }^{1}$ - D. S. Swetz ${ }^{1}$. \\ H. Tatsuno ${ }^{1}$ - L. R. Vale ${ }^{1}$ - J. N. Ullom ${ }^{1}$
}

Published online: 1 April 2016

(C) Springer Science+Business Media New York 2016

\section{Erratum to: J Low Temp Phys DOI 10.1007/s10909-015-1373-z}

In the original paper, we described two different architectures of a time-divisionSQUID multiplexer: a "mature" architecture (Section 2) and a "new" architecture (Section 3). For the new architecture, the stated value of the Joule-power dissipation of the multiplexer chip was incorrect.

The corrected last sentence of the first paragraph of Section 3 is as follows:

There is no SQ2 on the multiplexer chip. Per-column power dissipation in the multiplexer chip(s) is $1.8 \mathrm{nW}$. This value was determined via measurement of current and voltage during standard operation of the chips.

The online version of the original article can be found under doi:10.1007/s10909-015-1373-z.

W. B. Doriese

doriese@nist.gov

1 National Institute of Standards and Technology, Boulder, CO 80305, USA

2 Stanford University Department of Physics, Stanford, CA 94305, USA 